\title{
The equivalence of uninorms induced by the $U$-partial order
}

\author{
Emel Aşıc1 (D) \\ Department of Software Engineering, Faculty of Technology, Karadeniz Technical University, 61080 \\ Trabzon, Turkey
}

\begin{abstract}
In this paper, some properties of an order induced by uninorms are investigated. In this aim, the set of incomparable elements with respect to the $U$-partial order for any uninorm is introduced and studied. Also, by defining such an order, an equivalence relation on the class of uninorms is defined and this equivalence is deeply investigated. Finally, another set of incomparable elements with respect to the $U$-partial order for any uninorm is introduced and studied.
\end{abstract}

Mathematics Subject Classification (2010). 03E72, 03B52

Keywords. uninorm, partial order, equivalence

\section{Introduction}

Uninorms were introduced by Yager and Rybalov [27]. Uninorms are special aggregation operators which have proven to be useful in many applications like fuzzy logic, expert systems, neural networks, fuzzy system modeling [16, 18,26].

In recent years, the notation of the order induced by uninorms (nullnorms, triangular norms) has been studied widely. In this sense, in [22], $T$ - partial order, denoted $\preceq_{T}$, defined by means of t-norms on a bounded lattice has been introduced.

Based on these previous studies, in [1,17], respectively, $U$-partial order and $F$-partial order obtained from the uninorm and nullnorm have been introduced and some properties of these orders have been investigated.

The uninorms, nullnorms and t-norms were also studied by many other authors [2-4, 8-12,14, 15, 19-21, 24].

In the present paper, we introduce the set of incomparable elements with respect to the $U$-partial order for any uninorm on $[0,1]$. The main aim is to investigate some properties of this set. The paper is organized as follows. We shortly recall some basic notions in Section 2. In Section 3, we define the set of incomparable elements with respect to the $U$-partial order for any uninorm on $[0,1]$. Also, we determine the set of incomparable elements w.r.t. $U$-partial order for some special uninorms. So, we obtain general form for t-norms and t-conorms. Then, we define an equivalence relation on the class of uninorms on $[0,1]$. In section 4 , we define the set $\mathcal{J}_{U}^{(x)}$, consisting all incomparable elements with any $x \in(0,1)$ according to $\preceq_{U}$. Furthermore, we show that even if uninorms are equivalent

Email address: emelkalin@hotmail.com

Received: 14.10.2016; Accepted: 02.11.2017 
under this relation, it need not be the case that their partial orders coincide. Finally, we define and study another set of incomparable elements with respect to the $U$-partial order for any uninorm on $[0,1]$.

\section{Preliminaries}

Definition 2.1 ([25]). Let $(L, \leq, 0,1)$ be a bounded lattice. A triangular norm $T$ (briefly t-norm) is a binary operation on $L$ which is commutative, associative, non-decreasing in each variable and has neutral element 1 .

Definition $2.2([25])$. Let $(L, \leq, 0,1)$ be a bounded lattice. A triangular conorm $S$ (briefly t-conorm) is a binary operation on $L$ which is commutative, associative, non-decreasing in each variable and has neutral element 0 .

Example 2.3 ([23]). Well-known triangular norms and triangular conorms on $[0,1]$ are:

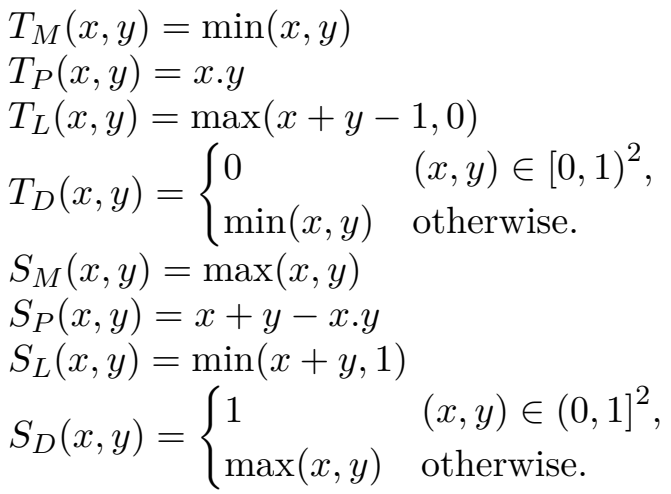

Extremal t-norms $T_{\wedge}$ and $T_{W}$ on $L$ are defined as follows, respectively:

$T_{\wedge}(x, y)=x \wedge y$

$T_{W}(x, y)= \begin{cases}x & \text { if } y=1, \\ y & \text { if } x=1, \\ 0 & \text { otherwise. }\end{cases}$

Similarly, the t-conorms $S_{\vee}$ and $S_{W}$ can be defined as above.

Especially we have obtained $T_{W}=T_{D}$ and $T_{\wedge}=T_{M}$ for $L=[0,1]$.

Definition $2.4([7])$. A t-norm $T$ on $L$ is divisible if the following condition holds:

$\forall x, y \in L \quad$ with $\quad x \leq y$ there is a $z \in L$ such that $x=T(y, z)$.

The infimum t-norm $T_{\wedge}$ is divisible: $x \leq y$ is equivalent to $x \wedge y=x$. A basic example of a non-divisible t-norm on an arbitrary bounded lattice $L$ (i.e., card $L>3$ ) is the t-norm $T_{W}$. Similarly, t-conorm $S_{\vee}$ is divisible. $S_{W}$ is a non-divisible t-conorm on an arbitrary bounded lattice $L$ (i.e., $\operatorname{card} L>3$ ).

Definition 2.5 ([6]). Let $(L, \leq, 0,1)$ be a bounded lattice. An operation $U: L^{2} \rightarrow L$ is called a uninorm on $L$, if it is commutative, associative, non-decreasing in each variable and has a neutral element $e \in L$.

We denote by $U(e)$ the set of all uninorms on $L$ with the neutral element $e \in L$. $A(e)=(0, e] \times[e, 1) \cup[e, 1) \times(0, e]$ for $e \in(0,1)$.

Definition 2.6 ([6]). A uninorm $U$ is called idempotent if $U(x, x)=x$ for all $x \in[0,1]$.

Definition 2.7 ([22]). Let $L$ be a bounded lattice, $T$ be a t-norm on $L$. The order defined as following is called a $T$ - partial order (triangular order) for t-norm $T$ :

$$
x \preceq_{T} y: \Leftrightarrow T(\ell, y)=x \text { for some } \ell \in L .
$$


Definition $2.8([17])$. Let $L$ be a bounded lattice, $S$ be a t-conorm on $L$. The order defined as following is called a $S$-partial order for t-conorm $S$ :

$$
x \preceq_{S} y: \Leftrightarrow S(\ell, x)=y \text { for some } \ell \in L .
$$

Definition $2.9([17])$. Let $(L, \leq, 0,1)$ be a bounded lattice and $U$ be a uninorm with neutral element $e$ on $L$. Define the following relation, for $x, y \in L$, as

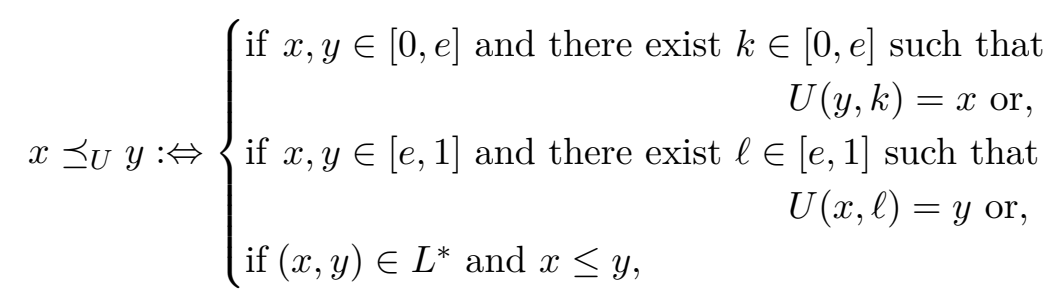

where $I_{e}=\{x \in L \mid x \| e\}$ and $L^{*}=[0, e] \times[e, 1] \cup[0, e] \times I_{e} \cup[e, 1] \times I_{e} \cup[e, 1] \times[0, e] \cup$ $I_{e} \times[0, e] \cup I_{e} \times[e, 1] \cup I_{e} \times I_{e}$.

Proposition 2.10 ([17]). The relation $\preceq_{U}$ defined in (2.1) is a partial order on L.

Proposition 2.11 ([13]). Let $T$ be a t-norm on $[0,1] . T$ is divisible if and only if $T$ is continuous.

\section{The set $K_{U} \subset[0,1]$ consisting of incomparable elements with respect to $\preceq_{U}$ on $[0,1]$}

In this section, we study the set of elements being incomparable with some other element with respect to the $U$-partial order $\preceq_{U}$ with some uninorm $U$ on $[0,1]$.

Let $U$ be a uninorm on $[0,1]$ and let $K_{U}$ be defined by

$$
\begin{array}{r}
K_{U}=\left\{x \in(0,1) \mid \text { for some } y \in(0,1),\left[x<y \text { and } x \npreceq_{U} y\right]\right. \\
\text { or } \left.\left[y<x \text { and } y \npreceq_{U} x\right]\right\} .
\end{array}
$$

Note that an element $x \in K_{U}$ is not necessarily incomparable with all elements $y \in$ $[0,1] \backslash\{0,1, x\}$.

We want to determine above introduced set for the smallest and greatest uninorms on $[0,1]$.

Proposition 3.1. Let $e \in[0,1]$. Consider the uninorm $\underline{U_{e}}:[0,1]^{2} \rightarrow[0,1]$ with neutral element e defined by

$$
\underline{U_{e}}(x, y)= \begin{cases}0 & (x, y) \in[0, e)^{2} \\ \max (x, y) & (x, y) \in[e, 1]^{2} \\ \min (x, y) & \text { otherwise. }\end{cases}
$$

Then, $K_{\underline{U_{e}}}=(0, e)$.

Proof. Let $x \in(0, e)$ and $y<x<1$. Let us show that $y \npreceq{\underline{U_{e}}}_{\underline{e}} x$. We consider $y{\underline{U_{e}}} x$. Then, there exists an element $\ell \in[0, e]$ such that $y=\underline{U_{e}}(\underline{x, \ell})$. Since $y \neq x$, it is not possible $\ell=e$. Since $(x, \ell) \in[0, e)^{2}$, it is obtained that

$$
y=\underline{U_{e}}(x, \ell)=0,
$$

a contradiction. Since for any $x \in(0, e)$, there exists an element $y<x<1$ such that $y \npreceq_{\underline{U_{e}}} x$. So, we have $x \in K_{\underline{U}_{e}}$. Thus, it is obtained that $(0, e) \subseteq K_{\underline{U_{e}}}$.

Conversely, let $x \in K_{U_{e}}$. We need to show that $x \in(0, e)$. Suppose that $x \notin(0, e)$. Since $x \in K_{\underline{U_{e}}}$, there exists an element $y \in(0,1)$ such that $x<y$ and $x \npreceq_{\underline{U_{e}}} y$ or $y<x$ and $y \npreceq_{U_{e}} x$. 
We assume that $e \leq x$.

- Let $x<y$ and $x \varliminf_{\underline{U}_{e}} y$.

Since $e \leq x$, it must be the case that $e<y$. Since $x<y$, we have $\max (x, y)=y$. By the definition of $\underline{U_{e}}$, we obtain that

$$
y=\max (x, y)=\underline{U_{e}}(x, y) .
$$

Then, it holds that $x{\underline{U_{e}}} y$, a contradiction.

- Let $y<x$ and $y \npreceq_{U_{e}} \bar{x}$. Since $e \leq x$, we have that $e<y$. Otherwise, if $y \leq e$, then it is obtained that $y \preceq_{\underline{U}_{e}} \bar{x}$, a contradiction by the definition of $\preceq_{U_{e}}$. Since $y<x$, it must be $\max (x, y)=x$. By the definition of $\underline{U_{e}}$, we obtain that

$$
x=\max (x, y)=\underline{U_{e}}(x, y) .
$$

Then, it holds that $y \preceq_{U_{e}} x$, a contradiction.

We consider that $x=0$. In this case, we have $0 \preceq_{U_{e}} y$, a contradiction. So, it must be the case that $x \in(0, e)$. Thus, it is obtained that $\overline{K_{U_{e}}} \subseteq(0, e)$. Consequently, we can show that $K_{\underline{U_{e}}}=(0, e)$.

Corollary 3.2. For the drastic product t-norm $T_{D}$ on $[0,1], K_{T_{D}}=(0,1)$.

Corollary 3.3. For the maximum t-conorm $S_{M}$ on $[0,1], K_{S_{M}}=\emptyset$.

Proposition 3.4. Let $e \in[0,1]$. Consider the uninorm $\bar{U}_{e}:[0,1]^{2} \rightarrow[0,1]$ with neutral element e defined by

$$
\overline{U_{e}}(x, y)= \begin{cases}\min (x, y) & (x, y) \in[0, e]^{2} \\ 1 & (x, y) \in(e, 1]^{2} \\ \max (x, y) & \text { otherwise. }\end{cases}
$$

Then, $K_{\bar{U}_{e}}=(e, 1)$.

Corollary 3.5. For the minimum $t$-norm $T_{M}$ on $[0,1], K_{T_{M}}=\emptyset$.

Corollary 3.6. For the drastic sum t-conorm $S_{D}$ on $[0,1], K_{S_{D}}=(0,1)$.

Lemma 3.7. Let $U$ be a uninorm on $[0,1]$. Then, $0 \preceq_{U} x, x \preceq_{U} x$ and $x \preceq_{U} 1$ for all $x \in[0,1]$.

Theorem 3.8. ([18]). Let $e \in[0,1] . U \in \mathcal{U}(e)$ if and only if

$$
U(x, y)= \begin{cases}T_{U} & (x, y) \in[0, e]^{2} \\ S_{U} & (x, y) \in[e, 1]^{2} \\ C & (x, y) \in A(e)\end{cases}
$$

where $T_{U}$ and $S_{U}$ are operations respectively isomorphic with some triangular norm and triangular conorm and increasing operation $C: A(e) \rightarrow[0,1]$ fulfills

$$
\min (x, y) \leq C(x, y) \leq \max (x, y) \text { for }(x, y) \in A(e) .
$$

Proposition 3.9. Let $U$ be a uninorm on $[0,1]$ with neutral element $e$ in Theorem 3.8. If $T_{U}$ and $S_{U}$ are continuous, then $K_{U}=\emptyset$.

Corollary 3.10. Let $e \in[0,1]$. Consider the uninorms $U^{\min }$ and $U^{\max }$ as unique idempotent uninorm $U_{e}^{\min }$ and $U_{e}^{\max }$, respectively:

$$
\begin{aligned}
U^{\min }(x, y) & = \begin{cases}\max (x, y) & (x, y) \in[e, 1]^{2}, \\
\min (x, y) & \text { otherwise. }\end{cases} \\
U^{\max }(x, y) & = \begin{cases}\min (x, y) & (x, y) \in[0, e]^{2}, \\
\max (x, y) & \text { otherwise. }\end{cases}
\end{aligned}
$$


Then, it is obtained that $K_{U^{\min }}=\emptyset$ and $K_{U^{\max }}=\emptyset$.

Theorem 3.11. ([18]). Let $U:[0,1]^{2} \rightarrow[0,1]$ be a uninorm with neutral element $\left.e \in\right] 0,1[$. Then the sections $x \mapsto U(x, 1)$ and $x \mapsto U(x, 0)$ are continuous in each point except perhaps for $e$ if and only if $U$ is given by one of the following formulas.

(a) If $U(0,1)=0$, then

$$
U(x, y)= \begin{cases}e T\left(\frac{x}{e}, \frac{y}{e}\right) & ,(x, y) \in[0, e]^{2} \\ e+(1-e) S\left(\frac{x-e}{1-e}, \frac{y-e}{1-e}\right) & ,(x, y) \in[e, 1]^{2} \\ \min (x, y) & ,(x, y) \in A(e),\end{cases}
$$

where $T$ is a $t$-norm and $S$ is a $t$-conorm.

(b) If $U(0,1)=1$, then the same structure holds, changing minimum by maximum in $A(e)$.

The class of uninorms as in case (a) will be denoted by $\mathcal{U}_{\min }$ and the class of uninorms as in case (b) by $\mathcal{U}_{\max }$. We will denote a uninorm $U$ in $\mathcal{U}_{\min }$ with underlying t-norm $T$, underlying t-conorm $S$ and neutral element $e$ by $U \equiv\langle T, e, S\rangle_{\min }$ and in a similar way, a uninorm in $\mathcal{U}_{\max }$ by $U \equiv\langle T, e, S\rangle_{\max }$.

Proposition 3.12. Let $U$ be a uninorm such that $U \equiv\langle T, e, S\rangle_{\min }$ or $U \equiv\langle T, e, S\rangle_{\max }$. Then,

$$
K_{U}=e K_{T} \cup\left(e+(1-e) K_{S}\right) .
$$

Proof. For any two elements $x, y \in[0, e]$, the $U$-comparability of $x$ and $y$ is equivalent to the $T$-comparability of $\frac{x}{e}$ and $\frac{y}{e}$. Therefore, $K_{U}$ contains the set $e K_{T}$. Similary, for any two elements $x, y \in[e, 1]$, the $U$-comparability of $x$ and $y$ is equivalent to the $S$-comparability of $\frac{x-e}{1-e}$ and $\frac{y-e}{1-e}$.

Therefore, $K_{U}$ contains the set $e+(1-e) K_{S}$.

For any two elements $x, y \in A(e)$, we have that $K_{U}=\emptyset$ by the definition of $\preceq_{U}$. On the other hand, if $(x, y) \in[0, e]^{2} \cup[e, 1]^{2}$, then proof is trivial. Also, if $(x, y) \in A(e)$, then proof is trivial by the definition of $\mathcal{U}_{\min }$ or $\mathcal{U}_{\max }$.

As an example of application of the previous proposition we consider $U=\left\langle T_{D}, e, S_{M}\right\rangle$ such that drastic product t-norm and maximum t-conorm. Then,

$$
K_{U}=(0, e) .
$$

Proposition 3.13. Let $U$ be a uninorm such that $U \equiv\langle T, e, S\rangle_{\min }$ or $U \equiv\langle T, e, S\rangle_{\max }$. Then,

i) If $x, y \in[0, e]$, then $x \preceq_{U} y$ if and only if $\frac{x}{e} \preceq_{T} \frac{y}{e}$.

ii) If $x, y \in[e, 1]$, then $x \preceq_{U} y$ if and only if $\frac{x-e}{1-e} \preceq_{S} \frac{y-e}{1-e}$.

Proof. (i)(Necessity) Let $x \preceq_{U} y$ for $x, y \in[0, e]$. Then, there exists an element $k \in[0, e]$ such that $U(y, k)=x$. By the definition of $U$, it must be the case that $x=e T\left(\frac{y}{e}, \frac{k}{e}\right)$. Then, we have $\frac{x}{e}=T\left(\frac{y}{e}, \frac{k}{e}\right)$. Since $\frac{k}{e} \leq 1$, it is obtained that $\frac{x}{e} \preceq_{T} \frac{y}{e}$.

(Sufficiency) Let $\frac{x}{e} \preceq_{T} \frac{y}{e}$ for $x, y \in[0, e]$. Then, there exists an element $\ell \in[0, e]$ such that $T\left(\frac{y}{e}, \ell\right)=\frac{x}{e}$, that is $x=e T\left(\frac{y}{e}, \ell\right)$. Clearly, $x=e T\left(\frac{y}{e}, \frac{\ell e}{e}\right)$. This means that $x=U(y, \ell e)$. Since $\ell e \leq e$, it is obtained that $x \preceq_{U} y$.

(ii) (Necessity) It can be shown that the case (i).

(Sufficiency) Let $\frac{x-e}{1-e} \preceq_{S} \frac{y-e}{1-e}$ for $x, y \in[e, 1]$. Then, there exists an element $\ell \in[e, 1]$ such that $S\left(\frac{x-e}{1-e}, \ell\right)=\frac{y-e}{1-e}$, that is $y=e+(1-e) S\left(\frac{x-e}{1-e}, \ell\right)$. It is clear that $y=e+(1-$ e) $S\left(\frac{x-e}{1-e}, \frac{(\ell-\ell e+e)-e}{1-e}\right)$. Since $e \leq \ell-\ell e+e$, it is obtained that $x \preceq_{U} y$.

$\preceq_{U}$-partial order introduced above allows us to introduce the next equivalence relation on the class of all uninorms on $[0,1]$. 
Definition 3.14. Define a relation $\beta$ on the class of all uninorms on $[0,1]$ by $U_{1} \beta U_{2}$,

$$
U_{1} \beta U_{2}: \Leftrightarrow K_{U_{1}}=K_{U_{2}} \text {. }
$$

Lemma 3.15. The relation $\beta$ given in Definition 3.14 is an equivalence relation.

Definition 3.16. For a given uninorm $U$ on $[0,1]$, we denote by $\bar{U}$ the $\beta$ equivalence class linked to $U$, i.e,

$$
\bar{U}=\left\{U^{\prime} \mid \quad U^{\prime} \beta U\right\} .
$$

Proposition 3.17. The set $[0,1] / \beta$, is uncountably infinite.

Proof. Let $e_{1}, e_{2} \in(0,1)$ and $e_{1} \neq e_{2}$. Suppose that $e_{1}<e_{2}$.

Consider the functions on $[0,1]$ defined as follows:

$$
\underline{U_{e_{1}}}(x, y)= \begin{cases}0 & (x, y) \in\left[0, e_{1}\right)^{2}, \\ \max (x, y) & (x, y) \in\left[e_{1}, 1\right]^{2} \\ \min (x, y) & \text { otherwise }\end{cases}
$$

and

$$
\underline{U_{e_{2}}}(x, y)= \begin{cases}0 & (x, y) \in\left[0, e_{2}\right)^{2} \\ \max (x, y) & (x, y) \in\left[e_{2}, 1\right]^{2} \\ \min (x, y) & \text { otherwise. }\end{cases}
$$

The functions $U_{e_{1}}$ and $U_{e_{2}}$ are uninorms on $[0,1]$ with neutral elements $e_{1}$ and $e_{2}$,

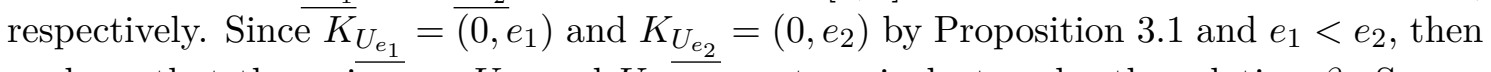
we have that the uninorms $\underline{U_{e_{1}}}$ and $\underline{U_{e_{2}}}$ are not equivalent under the relation $\beta$. So, we obtain that $\overline{U_{e_{1}}} \neq \overline{U_{e_{2}}}$.

Define the mapping $\delta:(0,1) \rightarrow[0,1] / \beta$ by

$$
\delta(e)=\underline{\overline{U_{e}}} .
$$

We showed that if $e_{1} \neq e_{2}$, then it must be $\delta\left(e_{1}\right) \neq \delta\left(e_{2}\right)$. So, $\delta$ is an injective function, it is obtained that $|(0,1)| \leq|[0,1] / \beta|$. So, the set $[0,1] / \beta$ has uncountably infinite cardinality.

Definition 3.18. Let $U_{1}$ and $U_{2}$ be two uninorms on $[0,1]$. If $\preceq_{U_{1}} \subseteq \preceq_{U_{2}}$, then we say that $U_{2}$ is order-stronger than $U_{1}$, or equivalently, that $U_{1}$ is order-weaker than $U_{2}$.

In [5], it was shown that for t-norms $T_{W}$ and $T_{\wedge}$ on $L, T_{W}$ is the order-weakest and $T_{\wedge}$ is the order-strongest t-norm, i.e., $\preceq_{T_{W}} \subseteq \preceq_{T} \subseteq \preceq_{T_{\wedge}}$. But for the uninorms, it need not be that case. Now, let us investigate the following example.

Example 3.19. Let us consider a smallest uninorm $\underline{U_{e}}:[0,1]^{2} \rightarrow[0,1]$ in Proposition 3.1 and a greatest uninorm $\bar{U}_{e}:[0,1]^{2} \rightarrow[0,1]$ in Proposition 3.4 with neutral elements $e$. We claim that $\underline{U_{e}}$ is not order-weakest and $\overline{U_{e}}$ is not order-strongest uninorm. We choose $e=\frac{1}{3}$. Since $\left.\underline{U_{e}} \overline{\left(\frac{1}{2}\right.}, \frac{2}{3}\right)=\frac{2}{3}$, it is obtained that $\frac{1}{2} \preceq_{\underline{U}_{e}} \frac{2}{3}$.

On the other hand, $\frac{1}{2} \npreceq \bar{U}_{U_{e}} \frac{2}{3}$. On the condition that $\frac{1}{2} \preceq_{\overline{U_{e}}} \frac{2}{3}$, there exists an element $\ell \geq \frac{1}{3}$ such that $\overline{U_{e}}\left(\frac{1}{2}, \ell\right)=\frac{2}{3}$. By the definition of $\overline{U_{e}}$, we have that $\overline{U_{e}}\left(\frac{1}{2}, \ell\right)=\min \left(\frac{1}{2}, \ell\right)=\frac{2}{3}$ or $\overline{U_{e}}\left(\frac{1}{2}, \ell\right)=\max \left(\frac{1}{2}, \ell\right)=\frac{2}{3}$. In first condition, we have $\ell=\frac{2}{3}$, a contradiction. In second condition, it is obtained that $\ell=\frac{2}{3}$ and $\left(\frac{1}{2}, \frac{2}{3}\right) \in(e, 1]^{2}$, a contradiction. So, $\frac{1}{2} \npreceq \overline{U_{e}} \frac{2}{3}$.

\section{About the set $\mathcal{J}_{U}^{(x)}$ consisting all incomparable elements with any $x \in$ $(0,1)$ according to $\preceq_{U}$}

Definition 4.1. Let $U$ be a uninorm on $[0,1]$ and let $\mathcal{J}_{U}^{(x)}$ for $x \in(0,1)$ be defined by

$$
\mathcal{J}_{U}^{(x)}=\left\{y \in(0,1) \mid\left[x<y \text { and } x \npreceq_{U} y\right] \text { or }\left[y<x \text { and } y \npreceq_{U} x\right]\right\} .
$$


After that the notation will be $\mathcal{J}_{U}^{(x)}$ to denote the set of all incomparable elements with $x \in(0,1)$ according to $\preceq_{U}$. The set $\mathcal{J}_{U}^{(x)}$ in Definition 4.1 can be defined on a bounded lattice and uninorm acting on this lattice.

We want to determine above introduced set for the smallest and greatest uninorms on $[0,1]$.

Proposition 4.2. Let us consider the smallest uninorm $\underline{U_{e}}$ with neutral element $e$ in Proposition 3.1. Then,

a) $\mathcal{J}_{U_{e}}^{(x)}=\{y \in(0, e) \quad \mid x \neq y\}$ for $x \in(0, e)$.

b) $\frac{\mathcal{J}_{\underline{U_{e}}}^{(x)}}{\underline{U_{e}}}=\emptyset$ for $e \leq x$.

a) Let $y \in(0, e)$ be arbitrary such that $x \neq y$ for $x \in(0, e)$. Let us show that $y \in \mathcal{J}_{\underline{U_{e}}}^{(x)}$. Suppose that $y \notin \mathcal{J}_{U_{e}}^{(x)}$. That is, $y$ is comparable to $x$ according to $\preceq_{U_{e}}$. Then, $y<x$ and $y \preceq_{U_{e}} x$ or $x<y$ and $x \preceq_{U_{e}} y$.

- Let $y<x$ and $y \preceq_{U_{e}} x$. Then there exists an element $k \in[0, e]$ such that $y=\underline{U}_{e}(x, k)$. Since $y \neq 0$, it must be case that

$$
y=\min (x, k)=\underline{U_{e}}(x, k)
$$

or

$$
y=\max (x, k)=\underline{U_{e}}(x, k) .
$$

If $y=\min (x, k)=\underline{U_{e}}(x, k)$, then we have that $y=k$ by $x \neq y$. In this case, we have that $y=0$, a contradiction from $x, y<e$. If $y=\max (x, k)=\underline{U_{e}}(x, k)$, then we have that $y=k$ by $x \neq y$. Then, we have that $y=0$, a contradiction from $x, y<e$. So, $y \in \mathcal{J}_{\underline{U_{e}}}^{(x)}$.

- Let $x<y$ and $x \preceq_{U_{e}} y$. Then, there exists an element $\ell \in[0, e]$ such that $x=U_{e}(y, \ell)$. Since $x \neq 0$, it must be the case that

$$
y=\min (x, k)=U(x, k)
$$

or

$$
y=\max (x, k)=U(x, k)
$$

and similar argument can be done for this case. So, $y \in \mathfrak{J}_{\underline{U_{e}}}^{(x)}$.

Conversely, let $y \in \mathfrak{J}_{U_{e}}^{(x)}$ be arbitrary for $x \in(0, e)$. By Lemma 3.7 it must be $x \neq y$. So, we need to show that $\overline{y \in}(0, e)$. Suppose that $y \notin(0, e)$. First, we assume that $y=0$. In this case $0 \preceq_{U_{e}} x$, a contradiction. So, it must be $y \neq 0$. Let $e \leq y$. Since $y \in \mathcal{J}_{\underline{U_{e}}}^{(x)}$, we have $y<x$ and $y \npreceq_{U_{e}} x$ or $x<y$ and $x \npreceq{\underline{U_{e}}} y$.

Let $y<x$ and $y \npreceq{\underline{U_{e}}} x$. Since $x=\max (x, y)$, by the definition of $\underline{U_{e}}$,

$$
x=\max (x, y)=\underline{U_{e}}(x, y) .
$$

It is obtained that $y \preceq{\underline{U_{e}}}_{\underline{e}} x$, a contradiction. Similarly, it can be shown that $x \npreceq_{\underline{U}_{e}} y$ for $x<y$. So, it must be the case that $y \in(0, e)$. Consequently, we can show that $\mathrm{J}_{\underline{U_{e}}}^{(x)}=\{y \in(0, e) \quad x \neq y\}$ for $x \in(0, e)$.

b) Let $e \leq x$. Suppose that $\mathcal{J}_{\underline{U_{e}}}^{(x)} \neq \emptyset$. Let $y \in \mathcal{J}_{\underline{U_{e}}}^{(x)}$ be arbitrary. So, it must be $y<x$ and $y \npreceq{\underline{U_{e}}} x$ or $x<y$ and $x \npreceq{\underline{U_{e}}}_{\bar{c}} \overline{\text {. }}$

Let $y<x$ and $y \npreceq_{\underline{U}_{e}} x$. If $y<e \leq x$, by the definition of $\preceq_{\underline{U}_{e}}$, it would be $y \preceq_{U_{e}} x$, a contradiction. If $e \overline{<y}<x$, then we have that $x=\max (x, y)$. $\overline{\mathrm{By}}$ the definition of $\bar{U}_{e}$, we have that $x=\max (x, y)=\underline{U_{e}}(x, y)$. It leads to $y \npreceq{\underline{U_{e}}} x$, a contradiction. Similarly it can be shown that $x<y$ and $x \npreceq_{\underline{U_{e}}} y$. So, $\mathcal{J}_{\underline{U_{e}}}^{(x)}=\emptyset$ for $e \leq x$. 
Proposition 4.3. Let us consider the greatest uninorm $\overline{U_{e}}$ with neutral element $e$ in Proposition 3.4. Then,

a) $\mathcal{J}_{\bar{U}}^{(x)}=\{y \in(e, 1) \quad \mid \quad x \neq y\}$ for $x \in(e, 1)$.

b) $\frac{\mathfrak{J}^{(x)}}{\bar{U}_{e}}=\emptyset$ for $x \leq e$.

Since we will use the results of the following example in subsequent propositions, it is useful to elaborate on the example.

Example 4.4. The uninorm $U:=U_{\min \left(T^{n M}, S_{M}, \frac{1}{2}\right)}:[0,1]^{2} \rightarrow[0,1]$ with neutral element $e=\frac{1}{2}$ defined as follows:

$$
U_{\min \left(T^{n M}, S_{M}, \frac{1}{2}\right)}(x, y)= \begin{cases}0 & (x, y) \in\left[0, \frac{1}{2}\right]^{2} \text { and } x+y \leq \frac{1}{2} \\ \max (x, y) & (x, y) \in\left[\frac{1}{2}, 1\right]^{2} \\ \min (x, y) & \text { otherwise. }\end{cases}
$$

Then,

a) $\mathcal{J}_{U}^{(x)}=\left\{y \in\left(0, \frac{1}{2}-x\right] \quad \mid \quad x \neq y\right\}$ for $x \in\left(0, \frac{1}{2}\right)$.

b) $\mathcal{J}_{U}^{(x)}=\emptyset$ for $\frac{1}{2} \leq x$.

Now, we want to show this claims.

a) Let $x<\frac{1}{2}$ and $y \in\left(0, \frac{1}{2}-x\right]$. Let us show that $y \in \mathcal{J}_{U}^{(x)}$. We assume that $y \notin \mathcal{J}_{U}^{(x)}$, i.e., $y<x$ and $y \preceq_{U} x$ or $x<y$ and $x \preceq_{U} y$.

Let $y<x$ and $y \preceq_{U} x$. Then, there exists an element $k \in\left[0, \frac{1}{2}\right]$ such that $y=U(x, k)$. Since $y \neq 0$, it must be the case that

$$
y=\min (x, k)=U(x, k) .
$$

Since $x \neq y$, it is obtained that $y=k$. Since $x, y<\frac{1}{2}$ and $y \in\left(0, \frac{1}{2}-x\right]$, it is obtained that $x+y \leq \frac{1}{2}$, a contradiction. Then, it must be the case that $y \in \mathcal{J}_{U}^{(x)}$.

Let $x<y$ and $x \preceq_{U} y$. Since $y \in\left(0, \frac{1}{2}-x\right]$, it is not possible the case $x<\frac{1}{2}<y$. So, $x<y<\frac{1}{2}$. Then, there exists an element $\ell \in\left[0, \frac{1}{2}\right]$ such that $x=U(y, \ell)$. Since $x \neq 0$, it must be

$$
x=\min (y, \ell)=U(y, \ell)
$$

and similar argument can be done for this case. So, $y \in \mathcal{J}_{U}^{(x)}$.

Conversely, let $y \in \mathcal{J}_{U}^{(x)}$. Suppose that $y \notin\left(0, \frac{1}{2}-x\right]$. Since $y \in \mathcal{J}_{U}^{(x)}$, we have $y<x$ and $y \npreceq_{U} x$ or $x<y$ and $x \npreceq_{U} y$. Let $y>\frac{1}{2}-x$.

Let $y<x$ and $y \npreceq_{U} x$. Since $y=\min (x, y)$ and $x+y>\frac{1}{2}$, by the definition of $U$, we have that

$$
y=\min (x, y)=U(x, y) .
$$

It is obtained that $y \preceq_{U} x$, a contradiction.

Let $x<y$. Similarly it can be shown that $x \npreceq_{U} y$.

If $y=0$, we have that $0 \preceq_{U} x$, a contradiction. So it must be $y \in\left(0, \frac{1}{2}-x\right]$. Consequently, we can show that $\mathcal{J}_{U}^{(x)}=\left\{y \in\left(0, \frac{1}{2}-x\right] \quad x \neq y\right\}$ for $x \in\left(0, \frac{1}{2}\right)$.

b) Let $\frac{1}{2} \leq x$. We assume that $\mathcal{J}_{U}^{(x)} \neq \emptyset$. Let $y \in \mathcal{J}_{U}^{(x)}$ be arbitrary. That is, $y<x$ and $y \npreceq_{U} x$ or $x<y$ and $x \npreceq_{U} y$.

Let $y<x$ and $y \npreceq_{U} x$. If $y<\frac{1}{2} \leq x$, by the definition of $\preceq_{U}$, it would be $y \preceq_{U} x$, a contradiction. If $\frac{1}{2}<y<x$, then we have that $x=\max (x, y)$. By the definition of $U$, we have that $x=\max (x, y)=U(x, y)$. It leads to $y \npreceq_{U} x$, contradiction. Similarly, if $x<y$ and $x \npreceq_{U} y$, then we have similar contradiction. So, it is obtained that $\mathcal{J}_{U}^{(x)}=\emptyset$ for $\frac{1}{2} \leq x$. 
Lemma 4.5. Let $U$ be a uninorm on $[0,1]$. Then $K_{U}=\bigcup_{x \in[0,1]} \mathfrak{J}_{U}^{(x)}$.

Proposition 4.6. Let $U_{1}$ and $U_{2}$ be two uninorms on $[0,1]$ with neutral element $e$. If $\preceq_{U_{1}} \subseteq \preceq_{U_{2}}$, then $\mathcal{J}_{U_{2}}^{(x)} \subseteq \mathcal{J}_{U_{1}}^{(x)}$ for all $x \in[0,1]$.

Proof. Let $U_{1}$ and $U_{2}$ be two uninorms on $[0,1]$ with neutral elements $e$ and $\preceq_{U_{1}} \subseteq \preceq_{U_{2}}$. We assume that $\mathcal{J}_{U_{2}}^{(x)} \nsubseteq \mathcal{J}_{U_{1}}^{(x)}$ for some $x \in(0,1)$. Let $y \in \mathcal{J}_{U_{2}}^{(x)}$ and $y \notin \mathcal{J}_{U_{1}}^{(x)}$ for some $y \in(0,1)$. Since $y \in \mathcal{J}_{U_{2}}^{(x)}$, then it must be case that $x<y, x \npreceq_{U_{2}} y$ or $y<x, y \npreceq U_{2} x$.

Without loss of generality, we assume that $x<y$ and $x \npreceq_{U_{2}} y$. Then, it must be $x<y<e$ or $e<x<y$. If $x<e<y$ or $y<e<x$, then we have that $x \preceq_{U} y$ or $y \preceq_{U} x$, a contradiction by the definition of $\preceq_{U}$. Without loss of generality, we assume that $x<y<e$ and $x \npreceq_{U_{2}} y$. Since $y \notin \mathcal{J}_{U_{1}}^{(x)}$ and $x<y$, it is obtained that $x \preceq_{U_{1}} y$. Since $\preceq_{U_{1}} \subseteq \preceq_{U_{2}}$, then we have that $x \preceq_{U_{2}} y$, i.e., $y \notin \mathcal{J}_{U_{2}}^{(x)}$, a contradiction. Thus, it is obtained that $\mathcal{J}_{U_{2}}^{(x)} \subseteq \mathcal{J}_{U_{1}}^{(x)}$ for all $x \in[0,1]$.

Remark 4.7. In Proposition 4.6, if $\preceq_{U_{1}} \subseteq \preceq_{U_{2}}$, then we can not say $\mathfrak{J}_{U_{1}}^{(x)} \subseteq \mathcal{J}_{U_{2}}^{(x)}$ for all $x \in[0,1]$. To illustrate this claim, the following example can be given:

Example 4.8. Consider the functions on $[0,1]$ defined as follows:

$$
U_{1}(x, y)= \begin{cases}0 & (x, y) \in\left[0, \frac{1}{2}\right)^{2} \\ 1 & (x, y) \in\left(\frac{1}{2}, 1\right]^{2} \\ y & x=\frac{1}{2}, \\ x & y=\frac{1}{2} \\ \min (x, y) & \text { otherwise }\end{cases}
$$

and

$$
U_{2}(x, y)= \begin{cases}\min (x, y) & (x, y) \in\left[0, \frac{1}{2}\right]^{2}, \\ \max (x, y) & \text { otherwise. }\end{cases}
$$

$U_{1}$ and $U_{2}$ are uninorms with neutral elements $\frac{1}{2}$ by [23]. It is clear that $\preceq_{U_{1}} \subseteq \preceq_{U_{2}}$. We claim that $\frac{1}{5} \in \mathcal{J}_{U_{1}}^{\left(\frac{1}{3}\right)}$ but $\frac{1}{5} \notin \mathcal{J}_{U_{2}}^{\left(\frac{1}{3}\right)}$. On the condition that $\frac{1}{5} \preceq_{U_{1}} \frac{1}{3}$, there exists an element $k \in\left[0, \frac{1}{2}\right]$ such that $U_{1}\left(\frac{1}{3}, k\right)=\frac{1}{5}$. If $k=\frac{1}{2}$, then we have $\frac{1}{5}=\frac{1}{3}$, a contradiction. If $k \in\left[0, \frac{1}{2}\right)$, then we have $\frac{1}{5}=0$, a contradiction. So, $\frac{1}{5} \npreceq_{U_{1}} \frac{1}{3}$. Thus, $\frac{1}{5} \in \mathcal{J}_{U_{1}}^{\left(\frac{1}{3}\right)}$. On the other hand, $\frac{1}{5} \notin \mathcal{J}_{U_{2}}^{\left(\frac{1}{3}\right)}$ by $U_{2}\left(\frac{1}{3}, \frac{1}{5}\right)=\frac{1}{5}$. We can generalize this set for the uninorms above as follows:

$$
\begin{aligned}
& \mathcal{J}_{U_{1}}^{(x)}=\left\{y \in(0,1) \quad \mid \quad x \neq y \text { and } y \neq \frac{1}{2}\right\} \text { for } x \in(0,1) \text { and } x \neq \frac{1}{2} . \\
& \mathcal{J}_{U_{2}}^{(x)}=\emptyset \text { for } x \in[0,1] .
\end{aligned}
$$

Corollary 4.9. Let $U_{1}$ and $U_{2}$ be two uninorms on $[0,1]$ with neutral elements $e$. If $\preceq_{U_{1}}=\preceq_{U_{2}}$, then $\mathcal{J}_{U_{1}}{ }^{(x)}=\mathcal{J}_{U_{2}}{ }^{(x)}$ for all $x \in[0,1]$.

Corollary 4.10. Let $U_{1}$ and $U_{2}$ be two uninorms on $[0,1]$ with neutral elements $e$. If $\preceq_{U_{1}}=\preceq_{U_{2}}$, then $K_{U_{1}}=K_{U_{2}}$.

Proposition 4.11. Let $U_{1}$ and $U_{2}$ be two uninorms on $[0,1]$. If for all $x \in[0,1], \mathcal{J}_{U_{1}}{ }^{(x)}=$ $\mathcal{J}_{U_{2}}{ }^{(x)}$, then the uninorms $U_{1}$ and $U_{2}$ are equivalent under the relation $\beta$.

Remark 4.12. The converse of Proposition 4.11 does not have to be true. Below is an example. 
Example 4.13. Let us consider a uninorm $U$ on $[0,1]$ in Example 4.4 and a uninorm $\underline{U}_{e}$ on $[0,1]$ in Proposition 3.1 with neutral elements $e=\frac{1}{2}$. By Proposition 3.1, we have $\frac{K_{U_{e}}}{K_{e}}\left(0, \frac{1}{2}\right)$. Now, we want to show $K_{U}=\left(0, \frac{1}{2}\right)$. Let $x \in\left(0, \frac{1}{2}\right)$ and $y \leq \frac{1}{2}$. By Example $4 . \overline{4}$, it is clear that $x<y, x \npreceq_{U} y$ or $y<x, y \npreceq_{U} x$. So, $x \in K_{U}$. That is, $\left(0, \frac{1}{2}\right) \subseteq K_{U}$. Conversely let $x \in K_{U}$ be arbitrary. We need to show that $x \in\left(0, \frac{1}{2}\right)$. Suppose that $x \notin\left(0, \frac{1}{2}\right)$. Since $x \in K_{U}$, then there exists an element $y \in(0,1)$ such that $x<y, x \npreceq_{U} y$ or $y<x, y \npreceq_{U} x$. Without loss of generality, we assume that $x<y, x \npreceq_{U} y$. If $x \geq \frac{1}{2}$, then we have that $y>x \geq \frac{1}{2}$. So, we have $\max (x, y)=y$. By the definition of $U$,

$$
U(x, y)=\max (x, y)=y .
$$

It is obtained that $x \preceq_{U} y$, a contradiction. If $x=0$, then we have $0 \preceq_{U} y$, a contradiction. So, it must be the case that $x \in\left(0, \frac{1}{2}\right)$. Thus, $K_{U} \subseteq\left(0, \frac{1}{2}\right)$. This proves that $K_{U}=\left(0, \frac{1}{2}\right)$. So, it is obtained that $K_{U_{e}}=K_{U}=\left(0, \frac{1}{2}\right)$. Thus, the uninorms $U$ and $\underline{U_{e}}$ are equivalent under the relation $\beta$. But, we claim that $\mathcal{J}_{U}{ }^{(x)}=\mathcal{J}_{\underline{U}_{e}}(x)$ for some $x \in(0,1)$.

Now, we want to show that this claim. Since $\frac{\overline{1}}{5}=U\left(\frac{2}{5}, \frac{1}{5}\right)$, we have that $\frac{1}{5} \notin \mathcal{J}_{U}{ }^{\left(\frac{2}{5}\right)}$. On the other hand, $\frac{1}{5} \npreceq_{U_{e}} \frac{2}{5}$. On the condition that $\frac{1}{5} \preceq_{U_{e}} \frac{2}{5}$, there exists an element $k \in\left[0, \frac{1}{2}\right]$ such that $\underline{U_{e}}\left(\frac{2}{5}, k\right)=\frac{1}{5}$. By the definition of $\underline{U_{e}}$, we have that $0=\frac{1}{5}=\underline{U_{e}}\left(\frac{2}{5}, k\right)$, a contradiction. So, $\frac{1}{5} \in \underline{J}_{\underline{U e}_{e}}{ }^{\left(\frac{2}{5}\right)}$. Thus, we have $\mathcal{J}_{U}{ }^{\left(\frac{2}{5}\right)} \neq \overline{\mathcal{J}}_{\underline{U}_{e}}{ }^{\left(\frac{2}{5}\right)}$.

Corollary 4.14. Although the uninorms $U_{1}$ and $U_{2}$ are equivalent under the relation $\beta$, it need not be the case that the $U_{1}$-partial order coincides with the $U_{2}$-partial order.

Definition 4.15. Let $U$ be a uninorm on $[0,1]$ with neutral element $e$. $K_{U}^{\star}$ is defined by

$$
\begin{gathered}
K_{U}^{\star}=\left\{x \in K_{U} \mid \text { for some } y, y^{\prime} \in(0, e),[x<y \text { but } x \npreceq U y]\right. \\
\text { and } \left.\left[y^{\prime}<x \text { but } y^{\prime} \npreceq_{U} x\right]\right\} .
\end{gathered}
$$

Remark 4.16. By the definition of $K_{U}^{\star}$, it is clear that $K_{U}^{\star} \subseteq K_{U}$. But the reverse inclusion may not be true. Now, let us investigate the following example.

Example 4.17. Let us consider a uninorm $U$ on $[0,1]$ with neutral element $e=\frac{1}{2}$ in Example 4.4. Since $K_{U}=\left(0, \frac{1}{2}\right)$ by Example 4.13, it is clear that $\frac{1}{4} \in K_{U}$. But $\frac{1}{4} \notin K_{U}^{\star}$. That is, there does not exist some $y \in(0,1), \frac{1}{4}<y$ and $\frac{1}{4} \npreceq_{U} y$. It is because that we have $\frac{1}{4}+y>\frac{1}{2}$ since $\frac{1}{4}<y$. So, we have that $U\left(y, \frac{1}{4}\right)=\frac{1}{4}$ by the definition of $U$. So, $\frac{1}{4} \preceq_{U} y$ for all $\frac{1}{4}<y$.

Remark 4.18. One can wonder which uninorms are provided $K_{U}^{\star}=K_{U}$. Consider the smallest uninorm $\underline{U}_{e}$ on $[0,1]$ with neutral element $e$ in Proposition 3.1. It can be shown that, for all $x \in(\overline{0, e})$, there exist elements $y, y^{\prime} \in(0, e)$ such that $x<y, x \npreceq_{U_{e}} y$ and $y^{\prime}<x, y^{\prime} \npreceq_{\underline{U_{e}}} x$. So, $K_{\underline{U_{e}}}^{\star}=(0, e)$. This show that $K_{\underline{U_{e}}}^{\star}=K_{\underline{U}_{e}}$. Also, consider the greatest uninorm $\overline{U_{e}}$ on $[0,1]$ with neutral element $e$ in Proposition 3.4. Similarly, $K_{\overline{U_{e}}}^{\star}=(e, 1)$. So, we have that $K_{\overline{U_{e}}}^{\star}=K_{\overline{U_{e}}}$.

The set, denoted $K_{U}^{\star}$, allows us to introduce the next equivalence relation on the class of all uninorms on $[0,1]$.

Definition 4.19. Define a relation $\beta^{\star}$ on the class of all uninorms on $[0,1]$ by $U_{1} \beta^{\star} U_{2}$,

$$
U_{1} \beta^{\star} U_{2}: \Leftrightarrow K_{U_{1}}^{\star}=K_{U_{2}}^{\star} \text {. }
$$

Lemma 4.20. The relation $\beta^{\star}$ given in Definition 4.19 is an equivalence relation.

Remark 4.21. Although $K_{U_{1}}=K_{U_{2}}$ for the uninorms $U_{1}$ and $U_{2}$, it need not be the case that $K_{U_{1}}^{\star}=K_{U_{2}}^{\star}$. To illustrate this claim we shall give the following example. 
Example 4.22. Let us consider a uninorm $U$ on $[0,1]$ in Example 4.4 and a uninorm $U_{e}$ on $[0,1]$ in Proposition 3.1 with neutral elements $e=\frac{1}{2}$. We know that $K_{U_{e}}=K_{U}=\left(0, \frac{\overline{1}}{2}\right)$. So, it is obtained that the uninorms $U_{e}$ and $U$ are equivalent under the relation $\beta$. Also, we have $K_{\underline{U_{e}}}^{\star}=\left(0, \frac{1}{2}\right)$ by Remark 4.18 . It is obtained that $K_{U}^{\star}=\left(0, \frac{1}{4}\right)$ by Example 4.17.

\section{Conclusion}

We have defined the set of incomparable elements with respect to the $U$-partial order for any uninorm on $[0,1]$. Also we have introduced and studied an equivalence relation $\beta$ defined on the class of all uninorms on $[0,1]$. We have defined that the set $\mathfrak{J}_{U}^{(x)}$, consisting all incomparable elements with any $x \in(0,1)$ accordingly to $\preceq_{U}$. Furthermore, we have shown that even if uninorms are equivalent under this relation, it need not be the case that their partial orders coincide. Finally, we have defined and studied another set of incomparable elements with respect to the $U$-partial order for any uninorm on $[0,1]$.

Acknowledgment. We are grateful to the anonymous reviewers and editors for their valuable comments which have enabled us to improve the original version of our paper.

\section{References}

[1] E. Aşıcı, An order induced by nullnorms and its properties, Fuzzy Sets Systs. 325, 35-46, 2017.

[2] E. Aşıc1, On the properties of the F-partial order and the equivalence of nullnorms, Fuzzy Sets Systs. 346, 72-84, 2018, doi: 10.1016/j.fss.2017.11.008.

[3] E. Aşıc1, Some notes on the F-partial order, in: Advances in Fuzzy Logic and Technology 2017, Kacprzyk J., Szmidt E., Zadroźny S., Atanassov K., Krawczak M. (eds), IWIFSGN 2017, EUSFLAT 2017, Advances in Intelligent Systems and Computing 641, 78-84, Springer, Cham, 2018.

[4] E. Aşıc1, On the migrativity property for uninorms and nullnorms, in: Information Processing and Management of Uncertainty in Knowledge-Based Systems, Medina J. et al. (eds), Theory and Foundations, IPMU 2018, Communications in Computer and Information Science 853, 319-328, Springer, Cham, 2018.

[5] E. Aşıcı and F. Karaçal On the T-partial order and properties, Inf. Sci. 267, 323-333, 2014.

[6] T. Calvo, B. De Baets and J. Fodor, The functional equations of Frank and Alsina for uninorms and nullnorms, Fuzzy Sets Systs. 120, 385-394, 2001.

[7] J. Casasnovas and G. Mayor, Discrete t-norms and operations on extended multisets, Fuzzy Sets Systs. 159, 1165-1177, 2008.

[8] G.D. Çayl, On a new class of t-norms and t-conorms on bounded lattices, Fuzzy Sets Systs. 332, 129-143, 2018, doi: 10.1016/j.fss.2017.07.015.

[9] G.D. Çaylı and P. Drygaś, Some properties of idempotent uninorms on a special class of bounded lattices, Inf. Sci. 422, 352-363, 2018.

[10] G.D. Çaylı and F. Karaçal, Construction of uninorms on bounded lattices, Kybernetika 53, 394-417, 2017.

[11] G.D. Çayll, F. Karaçal and R. Mesiar, On a new class of uninorms on bounded lattices, Inf. Sci. 367-368, 221-231, 2016.

[12] B. De Baets and R. Mesiar, Triangular norms on product lattices, Fuzzy Sets Systs. 104, 61-75, 1999.

[13] B. De Baets and R. Mesiar, Triangular norms on the real unit square, Proceedings of the 1999 EUSFLAT-ESTYLF Joint Conference, Palma de Mallorca, Spain, 351-354, 1999 . 
[14] J. Drewniak, P. Drygaś and E. Rak, Distributivity between uninorms and nullnorms, Fuzzy Sets Systs. 159, 1646-1657, 2008.

[15] P. Drygaś and E. Rak, Distributivity equation in the class of 2-uninorms, Fuzzy Sets Systs. 291, 82-97, 2016.

[16] D. Dubois and H. Prade, Fundamentals of Fuzzy Sets, Kluwer Academic Publishers, Boston 2000.

[17] U. Ertuğrul, M.N. Kesicioğlu and F. Karaçal, Ordering based on uninorms, Inf. Sci. 330, 315-327, 2016.

[18] J.C. Fodor, R.R. Yager and A. Rybalov, Structure of uninorms, Int. J. Uncertain. Fuzziness Knowl-Based Syst. 5, 411-427, 1997.

[19] S. Gottwald, A treatise on many-valued logic, Studies in Logic and Computation, Research Studies Press, Baldock, 2001.

[20] M. Grabisch, J.L. Marichal, R. Mesiar and E. Pap, Aggregation Functions, Cambridge University Press, 2009.

[21] F. Karaçal and E. Aşıcı, Some notes on T-partial order, J. Inequal. Appl. 2013, 219, 2013.

[22] F. Karaçal and M.N. Kesicioğlu, A T-partial order obtained from t-norms, Kybernetika 47, 300-314, 2011.

[23] E.P. Klement, R. Mesiar and E. Pap, Triangular Norms, Kluwer Academic Publishers, Dordrecht 2000.

[24] M. Mas, G. Mayor and J. Torrens, The distributivity condition for uninorms and t-operators, Fuzzy Sets Systs. 128, 209-225, 2002.

[25] S. Saminger, On ordinal sums of triangular norms on bounded lattices, Fuzzy Sets Systs. 157, 1403-1413, 2006.

[26] R.R. Yager, Uninorms in fuzzy system modeling, Fuzzy Sets Systs. 122, 167-175, 2001.

[27] R.R. Yager and A. Rybalov, Uninorm aggregation operators, Fuzzy Sets Systs. 80, 111-120, 1996. 